ARTIGOS 


\title{
SCIENCES PRATIQUES ET PRATIQUE DE LA SCIENCE
}

\author{
GILLES GASTON GRANGER
}

Le problème que nous nous proposons de formuler (1) est celui de l'association des sciences de l'homme à l'action. Toute science, il est vrai, depuis la grande prise de conscience du XVI siècle européen, semble bien se prolonger plus ou moins directement vers l'action. Mais le cas des sciences de l'homme est assurément très singulier, d'autant plus, sans doute, que la connaissance est en ces domaines moins assurée. C'est à préciser cette singularité que nous allons nous attacher, sans oublier pour autant que le philosophe ne saurait espérer tout au plus que rendre les difficultés plus patentes; c'est aux hommes de sciences qu'il appartient, s'il se peut, de les résoudre.

\section{LA CRISE DES "ANTI-SCIENCES"}

Depuis moins d'un lustre, une nouvelle critique qui se veut radicale produit - en France en tous cas - ses arguments et ses pamphlets sous l'invocation d'une "anti-science": anti-psychiâtrie, anti-économique, anti-psychanalyse (sous les espèces d'un anti-Oedipe): Il est élégant, on le sait, d'être nietzschéen par les temps qui courent. Mais quels qu'en soient l'inspiration ou le ton, cette critique articule au fond trois griefs majeurs, accusant les actuelles sciences de l'homme, premièrement d'être de simples facteurs de reproduction de la sociéte telle qu'elle est, - deuxièmement de pérenniser un cloisonnement sans fondement réel entre

(1) Nous rédigeons ici le canevas d'une communication faite aux Facultés Universitaires Saint-Louis, à Bruxelles, à l'occasion des journées d'études «Philosophie et sciences humaines» (29-30 mars 1974). 
diverses disciplines, - troisièmement de se complaire volontiers dans un formalisme mathématique illusoire. Griefs qu'on ne saurait traiter à la légère, et qui, à le bien prendre, renouvellent une objection constamment faite às ces disciplines, relativement à l'impossibilité d'une connaissance de l'homme neutre et positive, dénonçant alors une neutralité feinte pour lui substituer um engagement reconnu.

Dans l'état actuel des sociétés et des moeurs, la question devient pressante, le sociologue, l'économiste, le psychologue, le linguiste ayant été sommés de quitter leur cabinet. L'extension prodigieuse des moyens de vulgarisation a engendré une demande si puissante - et, doit-on le dire, si aveugle — qu'elle résiste à la disproportion pourtant évidente entre les espérances et les succès très modérés qu'obtiennent les techniques ou les rituels engendrés un peu hâtivement par leurs savoirs. Il est donc tout à fait sain de mettre en question cet appareil, de s'interroger sur la nature pratique des sciences de l'homme et d'en tirer si possible des conséquences quant à la portée et aux limites des connaissances qu'on en doit légitimement espérer.

Il n'est pas tout à fait inutile de rappeler que le statut d'une science empirique de l'homme en tant qu'animal raisonnable et politique a déjà été défini par Aristote, comme celui d'une science pratique, irréductible à la fois à une simple technique et à une theoria au sens strict. D'une certaine manière, et dans le contexte de la société grecque du IV. ${ }^{\circ}$ siècle, notre problème a donc trouvé une solution dont l'examen rapide est propre à nous mieux faire entendre le sens de nos difficultés.

Pour le Philosophe du Lycée, la science pratique est une connaissance des conditions de l'action bonne, du "bien-vivre" de l'homme en tant qu'homme. Mais la coupure entre science théorique et science pratique ne passe pas pour Aristote entre nature et culture, ni entre système causal, et système finalisé. La physique, science théorique, étudie en effet des systèmes finalisés, et certaines parties de l'oeuvre du Philosophe ébauchent une connaissance positive théorique des institutions et des actes de l'homme. La véritable distinction aristotélicienne nous paraît opposer une theoria comme connaissance des essences à une science pratique comme connaissance des genèses (lorsque le moteur est extérieur à l'être engendré) . 
Le problème d'une science pratique est donc posé dans l'aristotélisme en des termes qu'on ne pourrait retrouver aujourd'hui sans artifice, du fait, en particulier, que les natures, objets théoriques, y partagent avec l'acte et les produits humains le caractère de la finalité. Mais ce que pourrait nous enseigner encore valablement cette philosophie, c'est que les deux types de connaissance doivent être distingués d'abord non pas quant à leurs visées respectives, quant aux attitudes pragmatiques de contemplation ou d'intervention qu'on leur associe, mais essentiellement quant à la nature même de leurs objets, des types de réalité sur lesquels porte le savoir.

C'est dans cette perspective que nous pouvons chercher à préciser le sens de la critique ci-dessus invoquée. Nous ne l'entreprendrons pas toutefois sans mettre en garde contre les dangers d'une nouvelle Rhétorique de l'anti-science. Car nous ne sommes nullement disposés à cautionner, pour l'amour de la subversion pure, le dilemme qui nous contraindrait à déconsidérer toute tentative de connaissance objective des faits humains soit au profit d'un abandon à l'innocence supposée d'un âge d'or, soit au profit d'un recours à une doctrine totalitaire de l'homme présentée comme vérité universelle et sans appel.

\section{COMMENT SE CONSTITUENT LES CHAMPS DE CONNAISSANCE}

La première question que nous poserons d'abord peut être ainsi formulée: le découpage des faits est-il déterminé par la pratique?

Pour comprendre comment se produisent les problématiques en sciences humaines, il faut bien voir que la réalité vécue s'y présente directement comme symbolique. Sa signification est alors perçue de différentes manières au sein de groupes humains différents, et cette perception dégage des problèmes dont la position dépend largement de cette vision même. On pourra qualifier d'idéologique une telle constitution d'un champ de connaissance. On n'en sera pas moins requis de s'interroger sur le bien fondé d'une attitude épistémologique consistant à pretendre substituer les "vrais" problèmes aux questions "apparentes" ainsi formulées. Le philosophe en tous cas se doit d'enregistrer la transformation 
des problématiques tout aussi précisément que la transformation des faits, dans la mesure - fluctuante - où ceux-ci peuvent être constitués et appréhendés indépendamment des problématiques contemporaines associées. Le rôle essentiel de la réflexion critique, en l' occurence, sera de systématiser explicitement les perceptions du symbolique et d'en faire apparaître les présupposés.

A ce propos nous vient à l'esprit le mot célèbre de Marx: "L'humanité ne se pose jamais que les problèmes qu'elle peut résoudre". (Préface de la Critique de l'Économie politique, 1859). Si l'on entendait à la lettre cette affirmation, il faudrait admettre aussi bien dans les sciences de la nature que dans les sciences de l'homme, une sorte de développement harmonieux et continu; mais l'histoire nous montre la radicale inadéquation de cette image. Marx veut ici parler essentiellement des situations sociales, qui ne sont pensées comme conflictuelles qu'à partir du moment où les moyens de faire éclater le conflit existent: "Le problème lui-même ne surgit que là où les conditions matérielles pour le résoudre existent déjà, ou du moins sont en voie de devenir." Résoudre signifie évidemment ici décider d'un combat entre deux forces affrontées au profit de celle des deux qui est la plus neuve et la plus active. Il s'agit d'un problème d'action, à quoi du reste se ramène aux yeux de Marx tout problème de connaissance. Faut-il étendre le principe à toute question posée par les sciences de l'homme? Pour qui se permet de douter que le dogme de la lutte des classes fournisse en toute circonstance une réponse satisfaisante, le mot de Marx comporte encore néanmoins une interprétation que corrobore l'histoire des sciences: un problème - de connaissance objective - n'est véritablement posé que lorsque sa formulation rend sa solution possible. Et dans de nombreux cas, la solution entrevue permet une reformulation du problème qui conduit elle-même à une solution plus précise. Cette solidarité question-réponse n'est nullement spécifique des sciences de l'homme; mais elle y donne lieu à un couplage si serré et à une rétro-action si sensible, que la solution risque trop souvent de se résoudre en pétition de principe, la connaissance en tautologie.

Dans ces conditions, on voit que l'on ne peut sans précautions toutes particulières dire que la science des faits humains part d'un donné. Certains critiques de l'anti-science vont même jusqu'à présenter comme nouveau programme "une 
exploration des possibles", et assurer que les sciences de l'homme doivent "déboucher dans l'utopie" (Guillaume et Attali, in L'Anti-économique, Paris, 1974). Oui, si l'on entend par là que l'homme ne nous intéresse pas seulement comme nature, et quil nous faut garder de confondre des états, qui sont toujours à quelque degré conjoncturels, avec une situation fondamentale et éternelle. Mais une science de l' "utopie" n'est rien si elle ne cherche d'abord les possibles dans l'actuel. Elle ne peut se refuser, sous prétexte d'explorer les possibles, à étudier le fonctionnement des sociétés existantes, et ne peut déclarer sans portée des mécanismes du fait qu'ils soient intégrés à des situations particulières et transitoires .

$$
\text { * } \quad * \quad *
$$

Pour une appréciation correcte des problématiques, la seule règle justifié nous paraît être de maintenir une distinction et une tension entre la visée d'une explication des faits humains - par modèles locaux - et la visée philosophique d'intégration des faits dans une totalité, jamais accomplie par la science. Une connaissance scientifique de l'homme doit ainsi se garder de deux perversions opposées. L'une est celle d'un scientisme impérialiste qui se convertit en un dogmatisme métaphysique: il affirme qu'il y a une connaissance scientifique de la réalité humaine prise comme totalité (et en particulier de ses normes); mais cette connaissance est alors imposée sans contrôle véritable et la machinerie scientifique tourne à vide. L'autre est celle d'un pragmatisme sceptique qui refuse à la science toute possibilité de connaitre l'humain, et n'accepte d'elle que des recettes. On ne peut tenter d'éviter ces pièges qu'en se proposant une idée suffisamment claire de l'articulation de la connaissance du fait humain et de la pratique.

\section{CONNAISSANCE STRUCTURALE ET DECISION.}

Si l'on veut que le mot de science conserve un sens qui l'oppose à d'autres espèces de connaissance, et puisse continuer de s'appliquer aux sciences de la nature traditionnelles, il faut poser que toute science vise des objets. C'est-à-dire qu'elle ne peut opérer en tant que science que sur des abstractions, assorties de processus de contrôle convenablement définis, soit du point de vue d'une cohérence interne - qui dépend d'une certaine logique —, soit du point de vue de l'expérimentation - qui dépend d'une technique instrumentale. Le reproche de "formalisme stérile" fait à l'économique 
néo-classique, par exemple, ne dénonce pas un défaut absolu: l'appareil abstrait d'une science ne saurait être trop cohérent ni trop explicite; il porte en réalité sur le défaut de conceptualisation de son objet, qui apparaît comme trop grossier en tant que représentation contrôlable d'un phénomène, et par conséquent vainement raffiné au contraire en tant que structure abstraite.

Le succès de la connaissance scientifique requiert que soit satisfaite l'exigence d'une conceptualisation adéquate; l'accent mis aujourd'hui sur les vertus supposées du modèle masque aux yeux de certains les difficultés de la conceptualisation. On croit quelquefois que le piétinement des sciences de l'homme vient d'un défaut de technologie, et l'on dit que, peut-être, ni la sociologie, ni la psychologie, ni l'économique même, n'ont les mathématiques qu'elles méritent. Il faut poser le problème autrement, et dire que les sciences de l'homme n'ont pas encore découvert leurs concepts opératoires; la mathématique, comme l'Intendance, suivra, et l'on s'apercevra sans doute que dans certains cas elle aura même précédé.

Le besoin d' "interdisciplinarité", et le mythe qu'il crée, traduit, croyons-nous la prise de conscience progressive de cette insuffisance de conceptualisation. C'est un besoin de rompre avec la fixation traditionnelle du choix des variables stratégiques: à propos d'un même phénomène social, l'économiste, le sociologue, le psychosocioloque privilégieront spontanément des organisations différentes, et leurs discours seront très rapidement divergents. Or dans les sciences de la nature, le problème de l' "interdisciplinarité" s'est depuis longtemps posé, pour le physicien et le chimiste, par exemple. La solution a été la prise en considération de niveaux différents et l'opposition, au sens bachelardien, du "phénomène' au "noumène", rendue opératoire par des règles de passage: la structure électronique des atomes est le "noumène" des "phénomènes" de valence. Mas ni le sociologue, ni l'economiste ne savent encore articuler entre eux leurs savoirs.

La direction qui nous paraît être la plus féconde et la plus sûre pour une conceptualisation des faits humains conduit aux modèles que nous avons appelés ailleurs "cybernétiques". Dans de tels modèles apparaît un système-support, qualifié par analogie d'énergétique, et un système informa- 
tionnel, qui peut fournir, entre autre, une représentation interne plus ou moins complète ou déformée de l'ensemble du modèle. Ce système informationnel ne peut être schématisé comme enchevêtrement de forces, mais comme organisation de signifiants. Nous ne développerons pas ici cette proposition que nous avons exposée à diverses reprises ${ }^{(2)}$. Nous nous contenterons de souligner dans cette perspective les deux difficultés maîtresses qui font obstacle à son exécution.

La première concerne la description des données, qui doivent alors être recueillies a deux niveaux à la fois, énergétique et informationnel. Très souvent, ces deux niveaux se trouvent confondus dans les enquêtes empiriques. Aussi bien, une collecte utilisable des données informationnelles supposerait une analyse sémantique préalable du système, qui en fournirait les cadres. Mais quand cette analyse a été faite, ses résultats, au lieu d'avoir été intégrés comme étage informationnel dans un modèle cybernétique complet, ont été généralement interprétés comme constituant en eux-mêmes un modèle. C'est ainsi que la théorie des systèmes de parenté ou celle des structures mythiques ne sont susceptibles d'expliquer aucun fait, si on les considère en elles-mêmes. Elles décrivent des réseaux informationnels auxquels il faudrait adjoindre un schéma de soubassement énergétique pour obtenir un modèle se prêtant à une explication des faits.

Mais c'est ici que la seconde difficulté se présente: comment coupler la partie énergétique du modèle total à sa partie informationnelle? Comment "agissent" les "informations"? Sur le graphe d'une machine cybernétique les points de jonction du circuit informationnel et du circuit énergétique représentent des mécanismes que notre science sait produire et, peu ou prou, expliquer. Sur le graphe d'un modèle de fait humain, ces points sont encore pour nous, le plus souvent, des boîtes noires, dont la fonction de transition est même inconnue ou tout à fait incertaine. Dans chaque cas particulier, la recherche d'une solution au problème de l'articulation de l'informationnel à l'énergétique fait apparaître d'une façon concrète, et en même temps accessible à une connaissance contrôlable, le passage des structures aux événements, et débouche très naturellement sur une techno-

(2) par exemple recemment in Sobre el trato de los factos humanos como objetos, Dianoia, Mexico, 1973. 
logie de l'action. C'est ici, croyons-nous, le véritable lieu épistémologique d'une "théorie de la décision". Toute théorie de la décision tend vers une description de l'insertion de l'informationnel dans l'énergétique; en ce sens elle exprime bien autre chose qu'une simple orientation vers l'application, et si la rencontre d'un tel problème est inéluctable lorsqu'on s'efforce de constituer une connaissance scientifique des faits humains, on voit en quel sens non trivial il faut entendre le caractère pratique de cette science, et comment il est inscrit dans la nature même de l'objet.

\section{LES SCIENCES HUMAINES DANS LE CORPS SOCIAL.}

Nous terminerons par quelques brèves réflexions sur l'insertion des sciences de l'homme dans le corps social. Tout d'abord, qui utilise ces sciences, et comment? Sans aucun doute, les groupes dominants, qui ne sont pas seulement les groupes au pouvoir. Il faudrait s'interroger à ce propos sur le concept de "science de classe" dont le peu de consistance semble aujourd'hui tacitement reconnu, puisqu'il est de moins souvent allégué. Sans doute le choix des questions et des types de réponse est-il, comme nous le remarquions plus haut, étroitement dépendant d'une certaine perception de la réalité symbolique par des groupes plus ou moins étendus, parmi lesquels, tautologiquement, les groupes dominants dominent... Mais la question est de savoir si cette détermination pour ainsi dire naturelle des orientations de la connaissance, d'une part entraîne une fermeture du corps doctrinal constitué et un aveuglement durable à certains aspects de la réalité, - d'autre part, peut compenser la stérilité et l'inefficacité éventuelles de cette connaissance. Ce qui paraît importer pour l'histoire des sciences dans la dynamique des groupes sociaux, ce n'est pas à vrai dire que les intérêts de tel groupe soient représentés dans la science. C'est beaucoup plutôt que la structure sociale commande en partie l'inertie et la viscosité des paradigmes scientifiques.

La production des connaissances, dans le domaine des sciences humaines plus qu'ailleurs, est sans doute inséparable des utilisations escomptées. Une étude positive un peu complète de ces tentatives d'utilisation aurait donc beaucoup d'intérêt, mais elle est à peine esquissée et l'on peut craindre que l'entreprise ne rencontre de sérieux obstacles nés de l'amour-propre des utilisateurs et des producteurs. Sans 
préjuger de ces résultats, ce n'est pas trop s'avancer que de formuler deux réquisits pour que les sciences de l'homme puissent être effectivement utilisées par les "décideurs", et ne jouent pas seulement le rôle de discours parallèles aux opérations intuitives des hommes de l'art. Il faudrait en premier lieu qu'elles fournissent des modèles où les variables stratégiques soient bien définies et accessibles, et des modèles complets, auxquels ne manquent ni l'étage énergétique, ni l'étage informationnel. Il faudrait en second lieu qu'elles soient produites dans une société apte de par sa structure, à orienter sa propre action, condition qui n'est que très partiellement réalisée dans les sociétés contemporaines.

Pour qui croit pouvoir constater que de tels réquisits ne sont pas généralement satisfaits, l'enseignement des sciences de l'homme fait problème. Il nous paraît clair en tous cas que deux attitudes-refuges doivent être - et sont assez souvent - dénoncées: l'académisme, qui se cantonne dans l'exposé dogmatique de "connaissances" incertaines présentées comme assurées et acquises pour l'éternité, - le "philonéisme", qui se jette sans discernement sur toute nouveauté pour la mettre au rang de vérité confirmée. Au contraire, la voie la plus féconde dans les circonstances présentes, mais aussi la plus ingrate et la plus difficile, consisterait peut-être à suivre deux règles: premièrement, présenter les théories sans les détacher jamais de leur histoire et d'une critique conceptuelle, - deuxièmement, décrire les questions posées par la pratique sociale, et en particulier celles auxquelles ces théories ne font pas de place, ou qu'elles échouent manifestement à élucider. Ainsi serait-il permis d'espérer que l'enseignement éveille à la fois des utilisateurs conscients de nos ignorances, et des chercheurs disponibles pour l'invention d'une science future.

juillet 1974 . 